\title{
MECHATRONIC DEVICE FOR LOCOMOTOR TRAINING
}

\author{
Sławomir DUDA*, Damian GASIOREK*, Grzegorz GEMBALCZYK*, Sławomir KCIUK*, Arkadiusz MĘŻYK*
}

*Faculty of Mechanical Engineering, Department of Theoretical and Applied Mechanics, Silesian University of Technology, ul. Akademicka 2A, 44-100 Gliwice, Poland

Slawomir.Duda@polsı.pl, 522400@gmail.com, Grzegorz.Gembalczyk@polsl.pl, Slawomir.Kciuk@polsl.pl, Arkadiusz.Mezyk@polsl.pl

received 26 August 2015, revised 7 December 2016, accepted 9 December 2016

\begin{abstract}
This paper presents a novel mechatronic device to support a gait reeducation process. The conceptual works were done by the interdisciplinary design team. This collaboration allowed to perform a device that would connect the current findings in the fields of biomechanics and mechatronics. In the first part of the article shown a construction of the device which is based on the structure of an overhead travelling crane. The rest of the article contains the issues related to machine control system. In the prototype, the control of drive system is conducted by means of two RT-DAC4/PCl real time cards connected with a signal conditioning interface. Authors present the developed control algorithms and optimization process of the controller settings values. The summary contains a comparison of some numerical simulation results and experimental data from the sensors mounted on the device. The measurement data were obtained during the gait of a healthy person.
\end{abstract}

Key words: Mechatronic Device, Gait Reeducation, Rehabilitation, Real-Time Systems, Control Systems

\section{INTRODUCTION}

In the contemporary world, the number of traffic accidents and civilization-related diseases leading to the loss of motor abilities is constantly growing (Botticello et al., 2014). Numerous diseases and injuries may permanently affect this ability while hindering ordinary functioning of a person and often preventing one from performing daily activities that seem trivial to those in possession of full motor skills. Such a situation makes the patient dependent on the support of other persons and hinders autonomous performance of physiological activities or social functions. In case of occurrence of walking disorders, rehabilitation of the patient becomes a necessity. It is aimed at the recovery - as far as possible - of the lost motor abilities. This is related to retraining the patient's ability to walk in new circumstances (Bae and Tomizuka, 2012; Behrman and Harkema, 2000; Boyd and Little, 2005; Hesse and Werner, 2009; Mailah et al., 2009).

To meet the need to ensure fast and reliable rehabilitation leading to full recovery of abilities in patients with a walking dysfunction, various classes of instruments and devices are designed and manufactured. These include both the simplest ones and ones that are highly intricate and utilize state-of-the-art advancements. Currently, among all devices of this type, stabilometric and balance platforms equipped with handrails or a patient suspension system protecting the patient against fall are most commonly used in rehabilitation centres. Examples of such platforms are the Biodex and Tecno Body stabilometric and balance platforms (Sawers and Ting, 2014; Cao et al., 2014; Hussain et al., 2013; Sherafat et al., 2013).

The platform itself, however, does not make learning to walk possible. For that purpose, treadmills may be used, on which the patient - protected with a special harness system similar to the one employed in the platforms - may learn to move. The Gait Trainer by the Biodex company with an UWS OFFSET unloading system may serve as an example of such devices. Using this system, the patient supports himself using handrails or is suspended in a harness, while simultaneously moving on the treadmill (Mulroy et al., 2010; Walker, et al., 2010). In the recent years, devices additionally forcing the movement of lower limbs came into use as well. Examples of such devices are the Lokomat by Hocoma or AutoAmbulator by the HealthSouth company (Lunenburger et al., 2004). Such devices are also often used in rehabilitation and waking of persons in coma. The immobilization of pelvis, however, is an obstacle for conscious patients learning to walk. Studies have proved that the immobilisation of the pelvis while walking significantly changes its pattern (Akdogan and Adli, 2011; Hidler and Wall, 2005).

Additionally, the movement of the lower limb in these devices is limited only to the sagittal plane, without ensuring proper movements in the frontal or transverse planes. It is also not without significance that the movement on the treadmill is not the same as moving in space. Treadmill training introduces habits different from those assumed to be correct in walking. All this may lead to the strengthening of improper movements, which may cause arthritis or lead to future traumatic injuries. Devices such as the Gait Trainer by Rifton or Kaye Products constitute an attempt to depart from methods based on movement on a treadmill. The above are walking frames with wheels and a harness system for the patient. The possibility of the patient's movement in horizontal paths is a great advantage of this type of devices. As far as rehabilitation is concerned, however, there are some disadvantages: the immobilization of the pelvis, the use of seats for unloading and the impossibility to conduct such exercises as climbing the stairs (Marchal-Crespo and Reinkensmeyer, 2009).

Without a doubt, the most technologically advanced device 
that was recently introduced to the market is the ZeroG device patented in the United States (patent No. US 7,883,450 B2). ZeroG is a mechatronic system for the assistance of human movement with unloading, which allows for the movement of the subject along a given path. Depending on the current position of a human, resulting from the phase of walk, the system's drives provide a reaction, thus ensuring the necessary unloading value (Hidler et al., 2011).

Striving to meet the need for a modern, domestic rehabilitation device for persons with a walking impairment, the employees of the Department of Theoretical and Applied Mechanics of the Silesian University of Technology have undertaken to build a demonstrative device for the re-education of walk which allows for autonomous movement of the patient in unloading conditions within the framework of the NR03-0040-10 research and development programme. The completed project is innovative, as the conducted domestic and worldwide market analysis has demonstrated that no such device has been used in the rehabilitation of persons having problems with locomotion (Duda et al., 2011). A device that would connect the current findings in the fields of biomechanics and mechatronics. The application of the device in rehabilitation of persons with locomotion disorders, with underlying conditions of both traumatic and neurological nature, provides an improvement of the working conditions of the staff conducting the rehabilitation. Also the patient's safety is improved, as they will be able to perform walking-related exercises while being protected against fall and to perform the exercises in pre-set unloading conditions. An additional advantage over similar devices available in the market is the possibility to learn to climb the stairs and to cross obstacles that may be encountered in everyday life.

\section{CHARACTERISTICS OF THE DEVICE}

The following basic systems may be distinguished in the developed device (Duda et al., 2011):

- support frame made of aluminium profiles;

- mechanical elements of the drive systems;

- motors with controllers and a control cabinet;

- computer with real-time boards installed;

- sensors measuring the dynamic states of the device with power feed.

The structure of the device is based on the structure of an overhead travelling crane. As a result of installing three drives, the movement of the sling of the person in rehabilitation is possible in all axes of the Cartesian coordinate system. Fig. 1 presents the arrangement of the individual axes.



Fig. 1. Directions and senses of the axes of the global coordinate system corresponding to the movements of individual drives
The basic mechanical part of the device is constituted by drive systems responsible for the transfer of the movement from the motor to the working units. The proposed drive system has been presented in a structural form (Fig. 2).

The performance of all assumed objectives requires the control system to operate with different parameters, depending on the mode of operation. This is why it was decided to divide the tasks and performance of various operation modes into separate tasks. By means of special expansion boards, the Matlab/Simulink suite allows for real-time control and measurement data acquisition using real-time simulation methods. Real time operation is characterized by the maintenance of certain time regimes, due to which the solver's step of integration is kept with the real time (Campa et al., 2004; Faust et al., 2015).

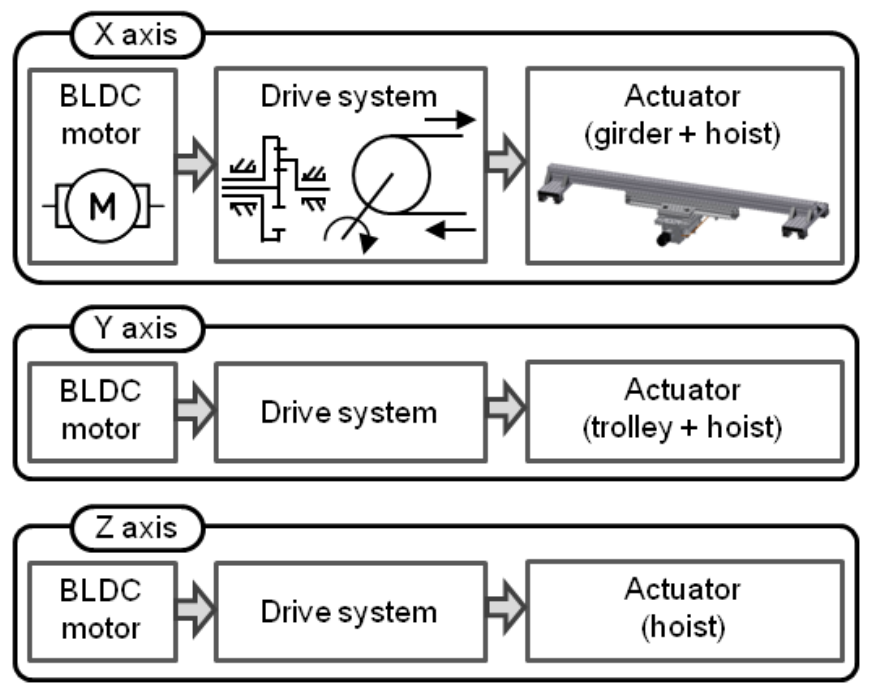

Fig. 2. Structural scheme of the drive system in mechatronic device for locomotor training

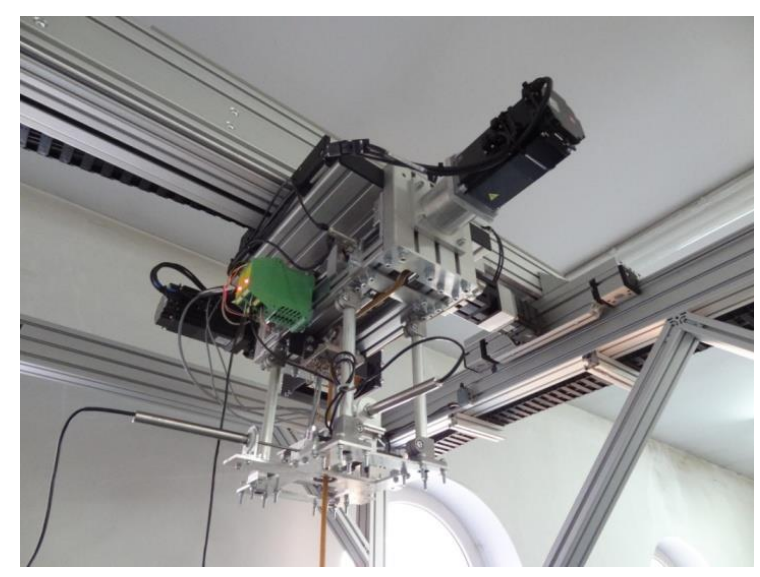

Fig. 3. The mechanism of dynamic unloading of patient with a rope displacement angle and force measurement system

The real-time systems connected to external devices are characterized by the fact that the calculations necessary for the supervision and control of the operations conducted by the controlled device are in parallel to the operation of the device. Moreover, the calculations give consideration to the current state of the device (Kaliński and Buchholz, 2015).

The transmission of control and measurement signals between the walking re-education device and the computer with the control system is conducted by means of two RT-DAC4/PCI real 
time boards connected with a signal conditioning interface. A control unit equipped with servo inverters of each of the motors is an additional element necessary for the correct operation of the device. As in each of the drives speed control is used, the servo inverter works as a regulator controlling the torque of the motor so as to ensure the required rotational speed (defined by the control signal). Each of the control signals is sent from real time boards to the control unit which works out the settings for the drive motors.

Analogue signals from the remaining sensors installed in the device are sent directly to the board conditioning the analogue signals. These sensors include the constructed devices for rope force measurement and the measurement of the sling rope deflection angle (Fig. 3) (Gembalczyk and Duda, 2012). The data transmission diagram has been presented in Figs. 4 and 5.

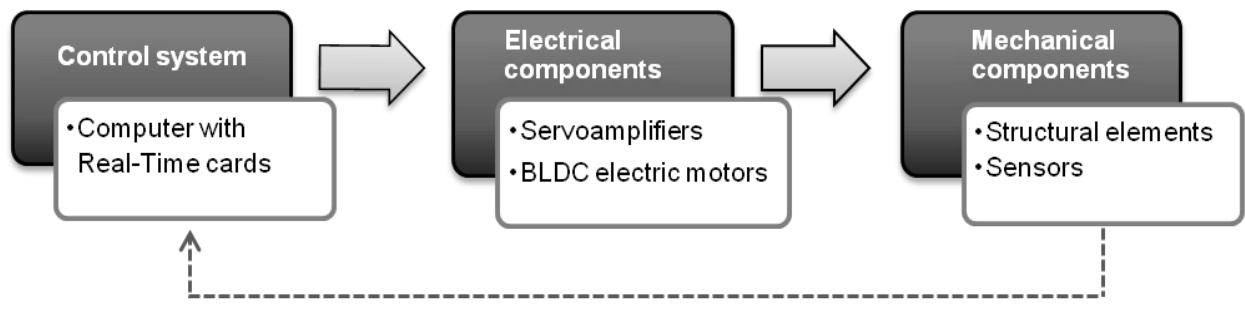

Fig. 4. Diagram of subassembly connections of the mechatronic device used in the process of walking reeducation

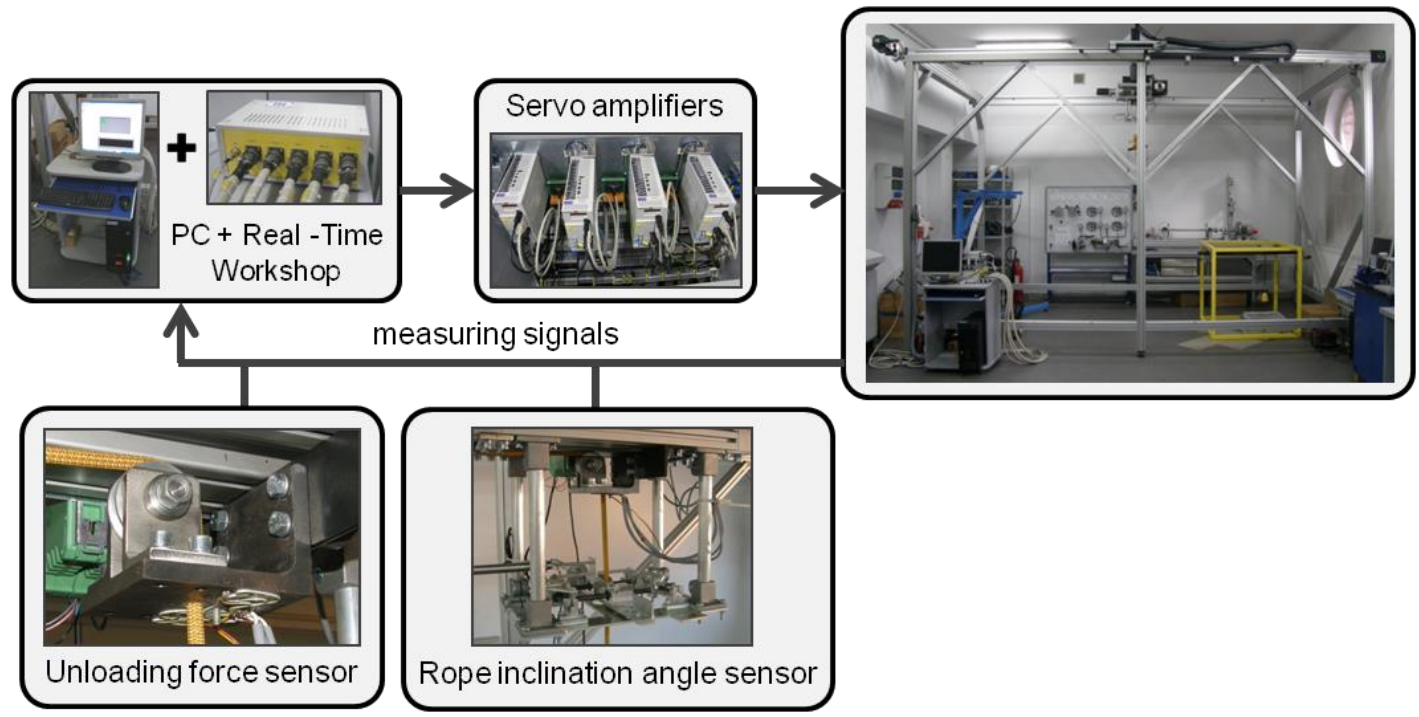

Fig. 5. Diagram of the control and measurement signal transmission

The control of the device is conducted by means of the MATLAB/Simulink suite. Two tabs are visualized in the main window of the application. Each of the tabs is responsible for the processing of different measurement, control and servomotor control signals. Due to the real-time library included in the MATLAB/Simulink software suite, the user of the programme may freely operate these signals (Duda et al., 2014).

\section{DRIVE CONTROL ALGORITHM}

The algorithm controlling the device's drives, encompassing the $X, Y$ and $Z$ axes, has been divided into three independent systems due to the expected performance method and the requirements of movement of the subassemblies. The control is performed in a closed control system using PD regulators by generating proper voltages which are supplied to BLDC motors.

A follow-up control system has been implemented for the $X$ and $Y$ axes. In this case, the algorithm of operation performs a certain wave of the controlled value, while the wave is not known. The purpose of this system is to control the object in such a way so as the changes of the regulated value would directly follow the changes of the pre-set value. In this case, the system aims at the minimization of the rope deflection angle. It is also possible to set a trajectory for the movement of the rope suspension point in the $X Y$ axis of the device (Duda et al., 2015).

For the $Z$ axis - the dynamic compensator of rope length, an algorithm aimed at keeping a constant force value in the rope has been implemented. This is necessary to achieve a constant unloading value required in some of the rehabilitation and walking re-education processes.

The correctness of the device operation, which is strictly related to the values of the regulators' settings, has been considered in the view of:

- ensuring the possibility of free movement - device directly following the patient,

- the possibility to set and maintain an assumed unloading level,

- ensuring the safety of the rehabilitated person - ensuring balance and preventing any possible falls.

In the proposed follow-up movement control system in the $X$ and $Y$ axes, the proper value of the rotational speed control signal of the drive motor is selected based on the misalignment signal. The misalignment signal equals the value of the crane rope de- 
flection from the perpendicular (the pre-set rope deflection angle value equals zero). This angle is dependent on the coordinates of the crane trolley and the location of the patient. The value of rope inclination angle is measured by a dedicated sensor that was designed and made by the authors of the article. The idea behind the algorithm controlling the follow-up movement of the crane has been presented in Fig. 6 .

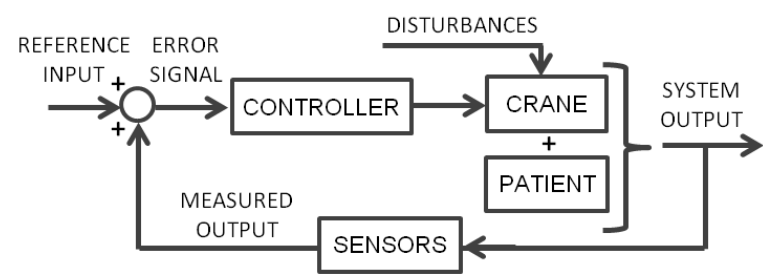

Fig. 6. Block diagram of the regulation system

To determine the values of the PD regulator settings, a numerical model of the tested device as a mechatronic system has been developed in the MATLAB/Simulink environment, giving consideration to complex couplings occurring between the individual elements of the system: the control unit - the drive - the human (Fig. 7). The numerical model allows also to calculate the rope deflection angle in $X Z$ and $Y Z$ planes $(\alpha, \beta)$ and the value of unloading force $\left(F_{\text {unl }}\right)$. The rotational speed control signals (CSX, CSy, CSz) of drive systems for each axis of the device are an inputs in the developed numerical model.

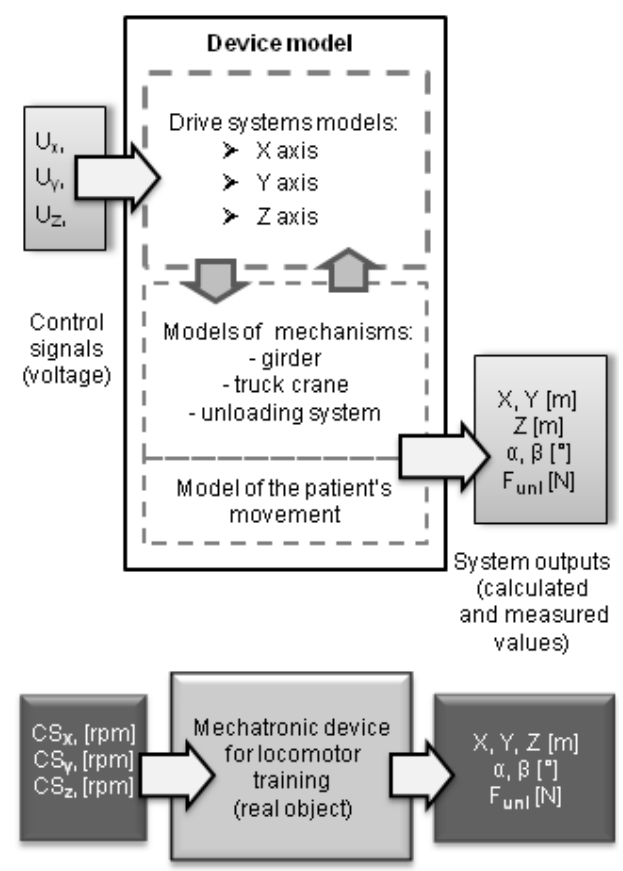

Fig. 7. Directions and senses of the axes of the global coordinate system corresponding to the movements of individual drives

The model of the patient's movement has been obtained from studies conducted with non-affected persons using a photogrammetric method (Cappozzo et al., 2005). In the initial selection of the setting values it has been noted that in case of excessive value of the $P$ proportional element, the trolley of the crane overtakes the moving person. This leads to a situation in which the suspension system starts to perform a reciprocating motion along the OX axis with increasing speed. This situation is presented in Figs. 8 and 9, exhibiting the movement of the suspension mechanism and the patient and the change in the value of the control signal of the rotational speed of the drive motor.

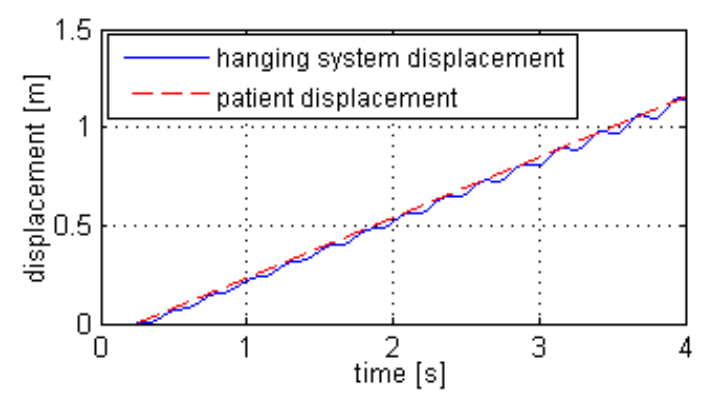

Fig. 8. Movement along $x$ axis of the patient and the crane trolley as a function of time

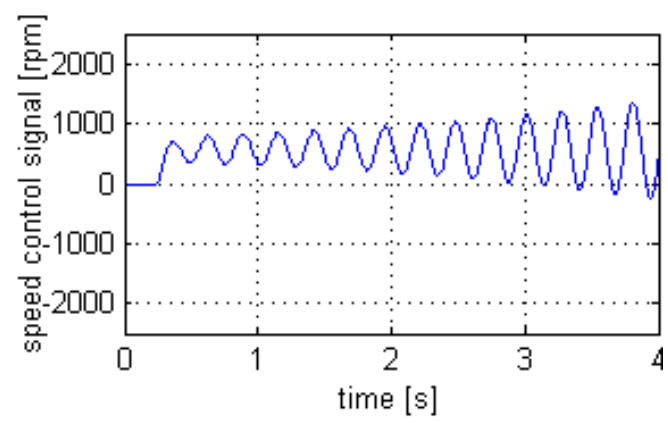

Fig. 9. Graph of the control signal of the rotational speed of the drive motor

The same phenomenon as in numerical simulations has been observed in the real object. An additional factor which affect on increasing such operation of the drive are the disturbances of signals from the measurement of the rope deflection angle, which constitute the feedback signal of the regulation system.

The conducted study has demonstrated that with excessive values of the PD regulator settings, the suspension system does not fluently perform the follow-up movement. Instead, high speed changes, especially notable during slow movement, occur during tracing. In the initial phase of the study, when the person moved with low speed (it is a follow-up movement, so the speed of the crane may be assumed to be approximately the patient's speed), the trolley would overtake the person and then conduct a reverse motion.

The presented situation causes both dangerous (potentially leading to a fall) and uncomfortable rehabilitation conditions. In selection of the optimal settings of the PD regulator, both the rope deflection angle and the changes of speed of the trolley of the suspension system during the movement of the patient were minimized (1) to ensure fluent operation at perpendicular orientation of the rope (in such position, the suspension system has the lowest impact on the natural movement of a person).

$F(\varphi) \cdot a+F(\ddot{\varphi}) \cdot b \rightarrow \min$

$F(\ddot{\varphi})=\int_{t=0}^{T}\left|\frac{d^{2} \varphi}{d t^{2}}\right| d t$

where: $\mathrm{T}$ - final time of simulation; $\mathrm{F}(\varphi)$ - function of the rope deflection in gait plane as a function of time; $F(\ddot{\varphi})$ - function describing the angular speed changes of the crane's rope in the 
direction of the patient's movement; $\mathrm{a}, \mathrm{b}$ - parameters specifying the impact of the deflection angle function and the linear speed change function on the minimized objective function.- description. The $a$ and $b$ parameters have been selected so that both functions (rope deflection angle and winch speed changes) would have a comparable impact on the objective function. With a problem formulated this way and assuming the patient's movement with a constant speed, the process of optimization of the regulator's parameters was conducted using a gradient method.
The resulting values $(\mathrm{P}=300, \mathrm{D}=10)$ have been implemented in the control system of the actual object. After the patient's movement registered in the tests was considered in the numerical model, the results of the simulation and experiment exhibited similar values. The functions presented in Figs. 10 constitute a comparison of the rope deflection angle in $X Z$ plane during the simulation and in the experiment. Figure 11 present the $x$-axis movement of the patient and the trolley registered during the tests of the actual object.

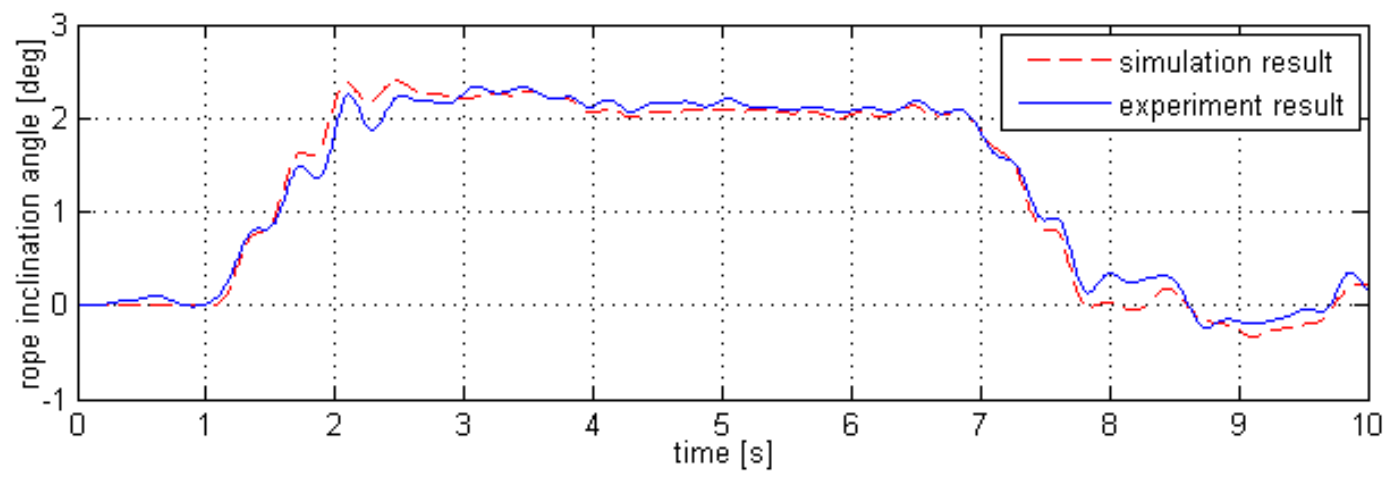

Fig. 10. Changes of the deflection angle of the rope of the crane sling in XZ plane as a function of time

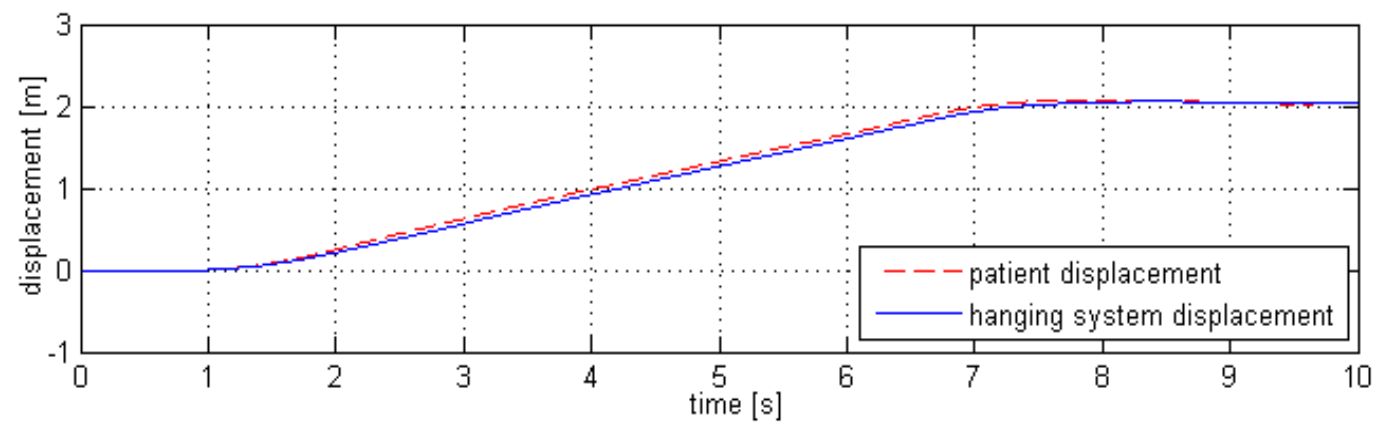

Fig. 11. Movement of the patient and the suspension system of the crane as a function of time

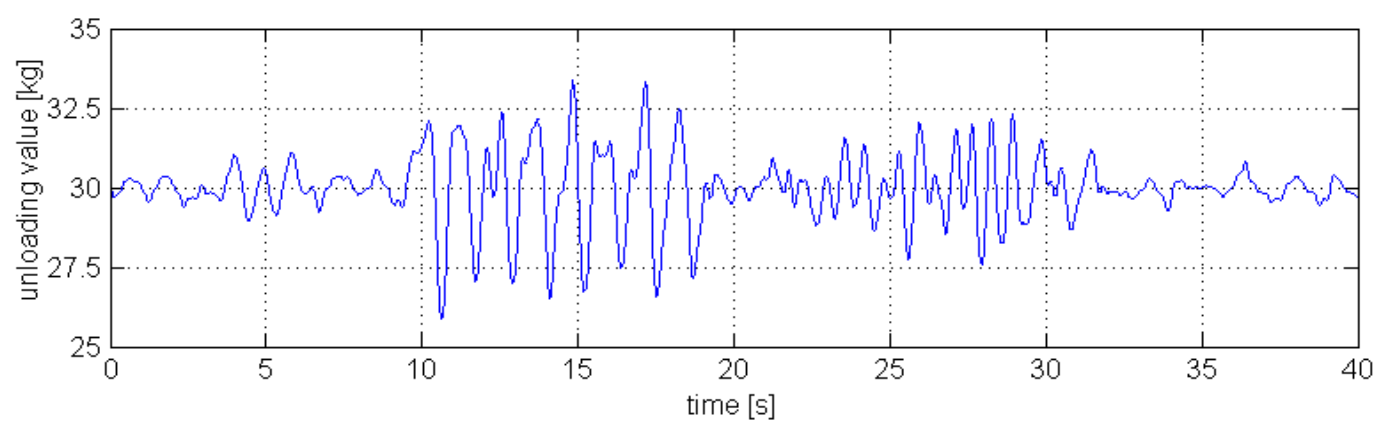

Fig. 12. Graph of the unloading force as a function of time

The parameters of the regulator responsible for the follow-up movement in the OY axis were selected in a similar manner. The obtained parameter values were after rounding: $\mathrm{P}=1100, \mathrm{D}=20$, respectively.

Based on the obtained results, it may be concluded that the used control algorithms of each of the axes function properly. It should be noted that in the device the $Y$ axis is a supportive axis, responsible for ensuring that the suspension mechanism follows the lateral movements of the patient. The main direction along which the patient is supposed to move is the direction of the X- axis.

When walking, the centre of inertia of a human moves along the perpendicular axis (the $Z$ axis of the device), which results in the changes of the force value in the rope on which the patient is suspended. This is why the control of the drive in the $Z$ axis is related to the maintenance of a constant, pre-set value of unloading force acting on the patient during the walk.

The selection of correct settings of the regulator has been conducted experimentally during a physical studies. This method of tuning the controller was caused by disturbances of the signal 
from the force measurement sensor. Excessive values of the proportional element in the regulator caused significant changes in the values of the control signal of the motor, which resulted in the instable operation of the motor and uncomfortable conditions of rehabilitation. The optimal value of the $P$ parameter selected in the algorithm amounted to 200. The graph of the unloading value during the test has been presented in Fig. 12.

\section{CONCLUSIONS}

The work presents the structure and real-time components cooperation in an innovative device used to support the rehabilitation process of persons affected by a walking impairment. The use of a computer as the management unit allows for the modification of algorithms and parameters of the control system, which may be motivated by the occurrence of new factors that were not considered earlier. Moreover, the continuous recording of the registered parameters obtained from the installed sensors was made possible, thus allowing for the assessment of rehabilitation progress.

The algorithms developed for the control of the device allow to conduct rehabilitation in two basic modes - as a protective followup system and in unloading conditions. Both modes were analyzed and tested, exhibiting the correctness of the proposed control algorithms.

The work presents a idea of computer tool for the testing of the dynamic behaviour of electric drives operating as a mechatronic device. The methodology of the conducted tests assumed the development of models for the individual systems and their further coupling in a loop to test whether the complete system works properly. The developed methodology allows for creating dynamics models for a wide range of rehabilitation devices which constitute mechatronic systems utilizing real-time control and their use in the design and the optimization of the structures of these devices and the synthesis of algorithms for controlling their movement.

\section{REFERENCES}

1. Akdogan E., Adli M.A. (2011), The design and control of a therapeutic exercise robot for lower limb rehabilitation: Physiotherabot, Mechatronics, 21(3), 509-522.

2. Bae J., Tomizuka M. (2012), A gait rehabilitation strategy inspired by an iterative learning algorithm, Mechatronics, 22(2), 213-221.

3. Behrman A.L., Harkema S.J. (2000), Locomotor training after human spinal cord injury: a series of case studies, Physical Therapy, 80(7), 688-700.

4. Botticello A.L., Rohrbach T., Cobbold N. (2014), Disability and the built environment: aninvestigation of community and neighborhood land uses and participation for physically impaired adults, Annals of Epidemiology, 24(7), 545-550.

5. Boyd J.E., Little J.J. (2005), Biometric gait recognition, Advanced Studies in Biometrics, Springer, 3161, 19-42.

6. Campa R., Kelly R., Santibanez V. (2004), Windows-based realtime control of direct-drive mechanisms: platform description and experiments, Mechatronics, 14(9), 1021-1036.

7. Cao J., Xie S.Q., Das R., Zhu G.L. (2014), Control strategies for effective robot assisted gait rehabilitation: The state of art and future prospects, Medical engineering \& Physics, 36(12), 1555-1566.
8. Cappozzo A., Della Croce U., Leardini A., Chiari L. (2005), Human movement analysis using stereophotogrammetry Part 1: theoretical background, Gait \& Posture, 21(2), 186-196.

9. Duda S., Gembalczyk G., Kciuk S., Gasiorek D. (2014), Mechatronic device to protect against falls during locomotor rehabilitation, Proceedings of the 3rd Joint International Conference on Multibody System Dynamics, Busan, 121-122.

10. Duda S., Kawlewski K., Gembalczyk G. (2015), Concept of the System for Control over Keeping up the Movement of a Crane, Solid State Phenomena, 220, 339-344.

11. Duda S., Michnik R., Kciuk S., Jurkojć J., Kawlewski K., Machoczek T. (2011), The conception of a mechatronic device for locomotor training, Aktualne Problemy Biomechaniki, 5, 29-36.

12. Faust O., Yu W., Acharya U.R. (2015), The role of real-time in biomedical science: A meta-analysis on computational complexity, delay and speedup, Computers in Biology and Medicine, 58, 73-84.

13. Gembalczyk G., Duda S. (2012), Design and validation of devices for measuring the force and the angle of inclination rope in crane, Modelowanie inżynierskie, 14(45), 32-38. [in Polish]

14. Hesse S., Werner C. (2009), Connecting research to the needs of patients and clinicians, Brain Research Bulletin, 78, 26-34.

15. Hidler J., Brennan D., Black I., Nichols D., Brady K. Nef T. (2011), ZeroG: Overground gait and balance training system, Journal of Rehabilitation Research \& Development, 48(4), 287-298.

16. Hidler J.M., Wall A.E. (2005), Alteration in muscle activation patterns during robotic-assisted walking, Clinical Biomechanics, 20, 184-193.

17. Hussain S., Xie S.Q., Jamwal P.K. (2013), Control of a robotic orthosis for gait rehabilitation, Robotics and Autonomous Systems, 61(9), 911-919.

18. Kaliński K.J., Buchholz C. (2015), Mechatronic design of strongly nonlinear systems on a basis of three wheeled mobile platform, Mechanical Systems and Signal Processing, 52-53, 700-721.

19. Lunenburger L., Colombo G., Riener R., Dietz V. (2004), Biofeedback in gait training with the robotic orthosis Lokomat, Engineering in Medicine and Biology Society, 4888-4891.

20. Mailah M., Jahanabadi H., Zain M.Z.M., Priyandoko G. (2009), Modelling and control of a human-like arm incorporating muscle models, Journal of Mechanical Engineering Science, 223(7), 1569-1577.

21. Marchal-Crespo L., Reinkensmeyer D.J. (2009), Review of control strategies for robotic movement training after neurologic injury, Journal of neuroengineering and rehabilitation, 6, 20.

22. Mulroy S.J., Klassen T., Gronley J.K., Eberly V.J., Brown D.A., Sullivan K.J. (2010), Gait parameters associated with responsiveness to treadmill training with body-weight support after stroke: an exploratory study, Physical Therapy, 90(2), 209-223.

23. Sawers A., Ting L.H. (2014), Perspectives on human-human sensorimotor interactions for the design of rehabilitation robots, Journal of neuroengineering and rehabilitation, 11, 142.

24. Sherafat S., Salavati M., Takamjani I.E., Akhbari B., Mohammadirad S., Mazaheri m., Negahban H. (2013), Intrasession and intersession reliability of postural control in participants with and without nonspecific low back pain using the Biodex Balance System, Journal of manipulative and physiological therapeutics, 36(2), 111-118.

25. Walker M.L., Ringleb S.I., Maihafer G.C., Walker R., Crouch J.R., Van Lunen B., Morrison S. (2010), Virtual reality-enhanced partial body weight-supported treadmill training poststroke: feasibility and effectiveness in 6 subjects. Archives of physical medicine and rehabilitation, 91(1), 115-122. 